\title{
Predictors of costs from the hospital perspective of primary pulmonary embolism
}

\author{
Serge Motte ${ }^{1,2}$, Christian Mélot $^{3}$, Lionel Di Pierdomenico ${ }^{1}$, Dimitri Martins ${ }^{1}$, \\ Pol Leclercq ${ }^{1}$ and Magali Pirson ${ }^{1}$
}

Affiliations: ${ }^{1}$ Health Economics, Hospital Management and Nursing Research Dept, School of Public Health, Université Libre de Bruxelles, Brussels, Belgium. ${ }^{2}$ Dept of Vascular Diseases, Erasme University Hospital, Université Libre de Bruxelles, Brussels, Belgium. ${ }^{3}$ Emergency Dept, Erasme University Hospital, Université Libre de Bruxelles, Brussels, Belgium.

Correspondence: Serge Motte, Dept of Vascular diseases, Hôpital Erasme, 808 Route de Lennik, 1070 Bruxelles, Belgium.

E-mail: serge.mottederasme.ulb.ac.be

ABSTRACT The objective of this study was to estimate the total hospital cost per patient admitted through the emergency department with a primary diagnosis of pulmonary embolism (PE), and to identify the main components and predictors of costs.

Actual costs of care of 652 consecutive patients hospitalised in 10 general hospitals in Belgium, including 31 outlier patients in terms of length of stay (4.8\%), were obtained by aggregating all cost components contributing to care of each patient.

In both inlier and outlier patients, the mean total cost per patient increased linearly with the degree of severity of illness classes related to the All Patient Refined Diagnosis Related Group $(\mathrm{p}<0.0001)$. Medical procedures, nursing activities and hospitalisation accommodation were the main cost components. We identified six independent predictors of costs in inliers: age group, chronic pulmonary heart disease, heart failure, admission to intensive care unit, initial thrombolysis treatment and type of hospital. There was a statistically significant linear trend between age groups and costs $(\mathrm{p}<0.0001)$

An increasing burden of comorbid illness was strongly associated with increasing actual cost for caring hospitalised patients for PE. Increasing age was associated with an increase in all main cost components.

@ERSpublications

Increased burden of comorbid illness is associated with increased cost of care for pulmonary embolism patients http://ow.ly/RSTPY

This article has supplementary material available from erj.ersjournals.com

Received: Feb 172015 | Accepted after revision: Aug 13 2015 | First published online: Oct 222015

Conflict of interest: None declared.

Copyright OERS 2016 


\section{Introduction}

Pulmonary embolism (PE) remains a major healthcare problem $[1,2]$. It presents with a variety of nonspecific symptoms and death occurs in $\sim 12 \%$ of cases within 1 month of diagnosis [2]. Recent studies have shown that the incidence of PE diagnosis assessed by analysing inpatient hospital discharge data is increasing with a concomitant increase in the use of computed tomography pulmonary angiography [3-5]. Analysis of inpatient hospital discharge minimal clinical summaries in Belgium shows that the total number per year of inpatients with a primary diagnosis of PE increased by $19 \%$ between 2000 and 2010 [6], the mean length of stay slightly decreased over the past decade, from 13 days in 2002 to 11 days in 2011, and the mean cost per patient borne by social security increased by $17 \%$ during the same period. These data are consistent with those reported in a recent study in Spain [4].

Few data exist on the actual costs from the hospital perspective for PE care. Most studies used reimbursement costs as an index of healthcare resource utilisation ([7-9] and https://tct.fgov.be/webetct/ etct-web/). However, actual costs may be significantly different from charges. Data regarding costs calculated by determining the use of all resources contributing to care of hospitalised patients with PE are scarce. The increase in the incidence of hospitalised PE patients with a concomitant increase in cost therefore raises the following issues: what may be the impact of the increase in cost on the budgets of the hospitals and what are the actual cost determinants?

The objective of this study was to evaluate patient-level costs estimated by using a hospital costing system and the types of hospital stay expenditures for patients admitted through the emergency department with a primary diagnosis of PE. A secondary objective was to identify independent determinants of costs.

Methods

Study group

The study was conducted in 10 general hospitals in Belgium. They were public and private urban nonteaching hospitals situated in the south part of Belgium. Their characteristics are presented in table 1.

This study was a retrospective analysis of inpatient hospital discharge minimal clinical summaries and cost records of consecutive inpatients admitted between January 1, 2008 and December 31, 2008 and January 1, 2010 and December 31, 2010. Minimal clinical summaries were included in the analysis if patients had been admitted via the emergency department and had a primary diagnosis of PE identified by using the International Classification of Diseases, Ninth Revision (Clinical Modification) (ICD-9-CM) codes 415.19 and 415.11.

Outlier patients in terms of length of stay were analysed separately with the aim of reducing the influence on the results of patients having an atypical length of stay. The following rule was used to define outlier patients: high outliers: 75 th percentile plus 1.5 times interquartile range (IQR); low outliers: 25th percentile minus 1.5 times IQR.

All inpatients had been categorised into similar disease groups by using the All Patient Refined Diagnosis Related Group (APR DRG) classification system (www.3m.com/product/information/All-Patient-RefinedDRG-Software). Within each diagnostic category, the classification system rates the severity of disease into minor, moderate, major or extreme severity of illness (SOI) or risk of mortality on the basis of the specific secondary diagnoses, and the interaction between secondary diagnoses, age, principal diagnosis and medical procedures. Secondary diagnoses were identified by using the following ICD-9-CM codes: active cancer $(148,151,152,153,154,157,162,174,180,183,185,189,190,197,198,199,202$, 203, 204, 239), concomitant

TABLE 1 Characteristics of the 10 hospitals for the year 2010

\begin{tabular}{lccc} 
Hospital & Beds & Total inpatients & $\begin{array}{c}\text { Total in- and outpatients } \\
\text { in the emergency department }\end{array}$ \\
\hline H1 & 307 & 12697 & 26599 \\
H2 & 419 & 15933 & 40084 \\
H3 & 288 & 14818 & 19000 \\
H4 & 10700 & 14598 \\
H5 & 317 & 9116 & 22017 \\
H6 & 324 & 20449 & 30298 \\
H7 & 535 & 17260 & 40292 \\
H8 & 362 & 10901 & 27321 \\
H9 & 278 & 9825 & 24520 \\
H10 & 317 & 13988 & 19858 \\
\hline
\end{tabular}

Data are presented as $n$. Hospital size: $\geqslant 350$ beds, $n=4 ;<350$ beds, $n=6$. 
deep venous thrombosis (DVT) $(451,453)$, history of previous venous thromboembolism (VTE) (V1251), chronic bronchitis (491), chronic pulmonary heart disease (416), chronic ischaemic heart disease (414, 412, V4581, V4582), heart failure (428), essential hypertension (401), diabetes mellitus (250), disorders of lipid metabolism (272) and asthma (493).

\section{Cost analysis}

The methodology used was a full costing method described elsewhere [10]. Briefly, the first step consisted of calculating the total costs (direct and indirect costs) of each activity centre (imaging, laboratory, hospital units, etc.). Direct costs were directly allocated to activity centres, whereas indirect costs were allocated in a first step to provisional cost centres and allocated in a second step to definitive activity centres using cost drivers (number of agents, work surfaces, number of inpatient days, etc.), by way of an allocation system based on the reciprocal allocation method. The second step was the allocation of the costs of each activity centre to medical production (e.g. physician costs of an imaging activity centre were allocated on medical procedures on the basis of three criteria (duration of the procedure, its risk and complexity) and nursing costs were allocated on the basis of minutes of nursing care). The third step consisted of calculating the cost of each patient. The principle was to determine how many cost drivers were consumed per stay. Cost drivers were, for example, medical procedures, drugs and minutes of nursing care. The cost of each patient was obtained by aggregating all cost drivers such as medical procedure costs, pharmaceutical costs and nursing costs consumed by each patient. Costs in 2008 were adjusted to reflect the increase of inflation in Belgium in 2010. New oral anticoagulants were not yet available in Belgium in 2010 for the treatment of PE.

\section{Statistical analysis}

As hospital payment systems in European countries have often been developed on the basis of average treatment costs of patients within a diagnosis-related group or on average length of stay as a proxy for costs, we calculated means, standard deviations and 95\% confidence intervals despite the skewness in distribution of costs and length of stay. Median with IQR (25th to 75th percentile) and proportions were calculated where indicated.

The variability of cost was assessed by calculation of the coefficient of variation (CV; ratio of the standard deviation to the mean $\times 100$ ) expressed as a percentage.

Age was discretised as age group pooled in six decades: $<40,40-49,50-59,60-69,70-79$ and $\geqslant 80$ years. ANOVA with polynomial contrast was used to assess linear (degree 1) and quadratic (degree 2) trends in log-transformed costs across age groups and SOI.

The association between cost as dependent variable and patient characteristics as independent variables was tested using univariable and multivariable linear regression analyses using the backward elimination technique. All variables with a $\mathrm{p} \leqslant 0.20$ were included in the multivariable analysis. Bootstrapping was used to compute the $95 \%$ confidence interval and to correct the coefficient for the bias using 1000 resamples (SPSS version 20; IBM, Chicago, IL, USA). Variables not available at admission were not included as potential predictors of cost. Predictors tested were age group, gender, concomitant DVT, history of previous VTE, chronic obstructive pulmonary disease (COPD), chronic pulmonary heart disease, chronic ischaemic heart disease including old myocardial infarction, coronary artery bypass graft or percutaneous transluminal coronary angioplasty, heart failure, hypertension, diabetes mellitus, disorders of lipid metabolism, and asthma. In addition to patient characteristics, the following possible independent variables were also included in the analyses: year of admission, type of hospital, initial thrombolysis treatment and admission to intensive care unit (ICU).

Costs were log-transformed in the regression analyses. The regression coefficients and corresponding 95\% confidence interval were exponential back-transformed to present the effect of individual determinants of costs as relative increase in cost (RIC) and corresponding 95\% confidence interval. $\mathrm{p}<0.05$ was considered significant.

\section{Results}

In total, 652 patients were identified from the hospital discharge minimal clinical summaries. Among these, 621 patients were inliers (95.2\%). Their demographic and clinical characteristics are presented in table 2. 382 inlier patients $(61.5 \%)$ were hospitalised in small hospitals with $<350$ beds. The mean length of stay was $9.98 \pm 6.09$ days. 31 patients were high outliers (4.8\%); their demographic and characteristics are presented in online supplementary table S1. There were no low outliers.

\section{Mean total cost}

The mean total cost per inlier patient was $4526 \pm 2996$ EUR. The mean log-transformed total costs per patient increased with the degree of SOI related to the APR DRG (ANOVA with polynomial contrasts showed a significant linear trend; $\mathrm{p}<0.0001)$. Among patients within the same SOI class, the costs varied 
TABLE 2 Characteristics of the 621 patients

$\begin{array}{lc}\text { Demographics } & 68 \pm 18 \\ \text { Age years } & 299(48.1) \\ \geqslant 75 \text { years old } & 237(38.2) \\ \text { Male } & \\ \text { Comorbidities to PE } & 232(37.4) \\ \text { Concurrent symptomatic DVT } & 66(10.6) \\ \text { Previous DVT or PE } & \\ \text { ICD-9-CM codes for secondary diagnoses } & 72(11.6) \\ \text { Active cancer } & 82(13.2) \\ \text { Chronic bronchitis } & 110(17.7) \\ \text { Chronic pulmonary heart disease } & 89(14.3) \\ \text { Chronic ischaemic heart disease } & 38(6.1) \\ \text { Heart failure } & 209(33.7) \\ \text { Essential hypertension } & 99(15.9) \\ \text { Diabetes mellitus } & 124(20.0) \\ \text { Disorders of lipid metabolism } & 14(2.3) \\ \text { Asthma } & \\ \text { APR DRG severity level } & 56(9.0) \\ \text { Minor } & 297(47.8) \\ \text { Moderate } & 209(33.7) \\ \text { Major } & 59(9.5) \\ \text { Extreme } & \end{array}$

Data are presented as mean \pm SD or $n$ (\%). PE: pulmonary embolism; DVT: deep venous thrombosis; ICD-9-CM: International Classification of Diseases, Ninth Revision (Clinical Modification); APR DRG: All Patient Refined Diagnosis Related Group.

substantially with a CV ranging from $46 \%$ to $56 \%$ (table 3). The median cost was 3866 (2567-5556) EUR and increased from SOI class 1 to 4 with a broad IQR range in all SOI classes.

The mean total cost per outlier patient was $14279 \pm 6915$ EUR. As for inliers, the mean log-transformed total costs per outlier patient increased with the degree of SOI related to the APR DRG (ANOVA with polynomial contrasts showed a significant linear trend; $\mathrm{p}<0.0001$ ) (online supplementary table S2). The median cost was 12992 (10295-15181) EUR.

\section{Cost components}

As far as the main cost components in inliers were concerned, medical procedures, nursing activities and hospitalisation accommodation represented $29 \%, 27 \%$ and $16 \%$, respectively, of the mean total cost per patient (table 4). Pharmaceutical products and administrative costs represented $8 \%$ and $7 \%$, respectively, of the total cost with a great dispersion of values observed in all degree of SOI classes, especially for pharmaceutical products (figure 1). In outlier patients, nursing activities, medical procedures and hospitalisation accommodation represented $34 \%, 22 \%$ and $20 \%$, respectively, of the mean total cost per patient (online supplementary table S3).

Predictors of cost in inlier patients

In univariable linear regression analysis, age groups, COPD, heart failure, essential hypertension, admission to ICU, initial thrombolysis treatment, year of admission and type of hospital were significantly associated with higher costs (table 5).

TABLE 3 Log-transformed total cost per patient according to the All Patient Refined Diagnosis Related Group SOI classes

\begin{tabular}{lccc} 
Sol class & Patients n & Mean (95\% Cl) cost EUR & Coefficient of variation \% \\
\hline Minor & 56 & $2264(1948-2631)$ & 51 \\
Moderate & 297 & $3246(3065-3437)$ & 46 \\
Major & 209 & $4364(4041-4713)$ & 51 \\
Extreme & 59 & $7598(6350-9091)$ & 56
\end{tabular}

SOI: severity of illness. " : log costs were de-transformed with $95 \%$ confidence interval. ANOVA with polynomial contrasts showed a significant linear trend for SOI ( $p<0.0001)$. 
TABLE 4 Cost components according to the All Patient Refined Diagnosis Related Group SOI class

Cost components

SOl class

All patients

\begin{tabular}{lccccc}
\cline { 2 - 4 } & Minor & Moderate & Major & Extreme & \\
\hline Nursing activities & $635 \pm 413$ & $969 \pm 603$ & $1353 \pm 830$ & $2565 \pm 1669$ & $1220 \pm 959$ \\
Medical procedures & $912 \pm 475$ & $1123 \pm 545$ & $1426 \pm 701$ & $2327 \pm 1210$ & $1320 \pm 773$ \\
Pharmaceutical products & $156 \pm 221$ & $230 \pm 315$ & $367 \pm 359$ & $1021 \pm 1269$ & $345 \pm 547$ \\
Hospitalisation accommodation & $399 \pm 114$ & $587 \pm 294$ & $829 \pm 485$ & $1317 \pm 730$ & $721 \pm 479$ \\
Administrative costs & $197 \pm 114$ & $253 \pm 136$ & $325 \pm 165$ & $540 \pm 316$ & $299 \pm 190$
\end{tabular}

Data are presented as mean \pm SD. SOI: severity of illness.

In multivariable linear regression analysis, we identified six independent predictors of costs: age group (RIC 1.07; 95\% CI 1.04-1.10), chronic pulmonary heart disease (RIC 1.18; 95\% CI 1.07-1.29) heart failure (RIC 1.52; 95\% CI 1.25-1.85), admission to ICU (RIC 1.49; 95\% CI 1.31-1.69), initial thrombolysis treatment (RIC 1.54; 95\% CI 1.27-1.88) and type of hospital (RIC 1.06; 95\% CI 1.04-1.07). A RIC of 1.07 for patient's age means that costs were increased by $7 \%$ per every 10 years of age starting at age $<40$ years (table 6). The addition of the 31 outliers in the analyses did not change the multivariable model (online supplementary table S4).

The analysis of the influence of age on the cost components is shown in table 7. There was a statistically significant linear trend between age groups and mean costs of nursing activities, medical procedures, hospitalisation accommodation and administrative costs. Conversely, costs for pharmaceutical products did not increase systematically with age groups.

\section{Discussion}

Few data exist on the actual costs for PE care. Most studies focused on charges as an index of healthcare resource utilisation [3, 4, 7-9]. However, charges may significantly differ from actual costs. They do not represent a proper estimation of healthcare resource utilisation and do not reflect cost differences between patients [11]. In the present study, the actual cost from the hospital perspective of each patient stay was obtained by aggregating all cost drivers contributing to the care of each patient. Patients with higher SOI levels had higher costs compared with patients with low SOI, suggesting that more severe comorbidities as measured by the APR DRG SOI were associated with a significant increase in cost. SOI levels are calculated using an algorithm developed by $3 \mathrm{M}$ and marketed as a software package (see Methods section). These levels stratify patients according to the extent of organ system loss of function or physiological decompensation, and are based on the interaction between secondary diagnoses, age, principal diagnosis and procedures. Other studies in different clinical settings have shown the ability of APR DRG SOI stratification to predict resource utilisation from the healthcare system perspective [12-16].

As the methodology used in the present study valued all relevant hospital services at the most detailed level per individual patient, our cost estimates reflect the variability of care in a real-world setting as

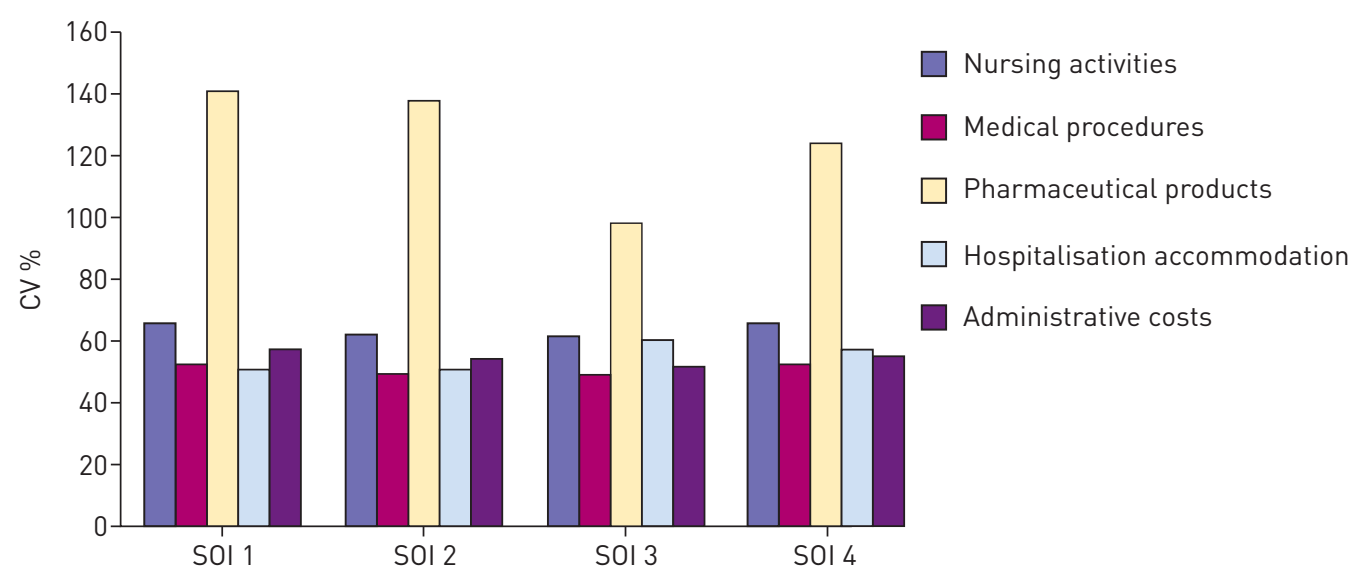

FIGURE 1 Variability of main cost components according the All Patient Refined Diagnosis Related Group severity of illness (SOI) class assessed by calculation of the coefficient of variation (CV). 
TABLE 5 Univariable linear regression analysis of predictors of log-transformed total cost

\begin{tabular}{|c|c|c|c|}
\hline Variable & Patients n & Mean $(95 \% \mathrm{Cl})$ total cost per stay EUR & p-value \\
\hline \multicolumn{4}{|c|}{ Age group years } \\
\hline$<40$ & 54 & $3131(2681-3581)$ & \\
\hline $40-49$ & 65 & $4008(3394-4622)$ & $0.099^{\#}$ \\
\hline $50-59$ & 52 & $4167(3162-5173)$ & $0.549^{\#}$ \\
\hline $60-69$ & 100 & $4090(3501-4679)$ & $0.310^{\#}$ \\
\hline $70-79$ & 157 & $5151(4665-5636)$ & $<0.001^{\#}$ \\
\hline$\geqslant 80$ & 193 & $4905(4473-5337)$ & $<0.001^{\#}$ \\
\hline \multicolumn{4}{|c|}{ ANOVA with polynomial contrasts for linear trend $p<0.0001$} \\
\hline \multicolumn{4}{|c|}{ Concurrent symptomatic DVT } \\
\hline Yes & 233 & 4604 (4230-4978) & 0.558 \\
\hline No & 388 & $4479(4174-4784)$ & \\
\hline \multicolumn{4}{|c|}{ Previous DVT or PE } \\
\hline Yes & 67 & $4412(3687-5136)$ & 0.618 \\
\hline No & 554 & $4540(4289-4790)$ & \\
\hline \multicolumn{4}{|l|}{ Gender } \\
\hline Male & 237 & 4437 (4041-4832) & 0.338 \\
\hline Female & 384 & $4581(4286-4876)$ & \\
\hline \multicolumn{4}{|c|}{ Active cancer } \\
\hline Yes & 72 & 4548 (3797-5299) & 0.780 \\
\hline No & 549 & 4523 (4274-4772) & \\
\hline \multicolumn{4}{|c|}{ Chronic pulmonary heart disease } \\
\hline Yes & 110 & 4750 (4214-5285) & 0.178 \\
\hline No & 511 & 4478 (4214-4741) & \\
\hline \multicolumn{4}{|c|}{ Chronic ischaemic heart disease } \\
\hline Yes & 89 & $4843(4245-5441)$ & 0.133 \\
\hline No & 532 & $4473(4216-4730)$ & \\
\hline \multicolumn{4}{|l|}{ COPD } \\
\hline Yes & 80 & $5489(4606-6373)$ & 0.017 \\
\hline No & 541 & $4384(4147-4620)$ & \\
\hline \multicolumn{4}{|c|}{ Heart failure } \\
\hline Yes & 37 & 7770 (6092-9449) & $<0.001$ \\
\hline No & 584 & $4320(4101-4540)$ & \\
\hline \multicolumn{4}{|c|}{ Essential hypertension } \\
\hline Yes & 209 & $4719(4320-5117)$ & 0.043 \\
\hline No & 412 & 4428 (4134-4722) & \\
\hline \multicolumn{4}{|c|}{ Diabetes mellitus } \\
\hline Yes & 95 & 5091 (4373-5810) & 0.107 \\
\hline No & 526 & $4434(4177-4671)$ & \\
\hline \multicolumn{4}{|c|}{ Disorders of lipid metabolism } \\
\hline Yes & 124 & 4670 (4203-5138) & 0.156 \\
\hline No & 497 & $4490(4218-4762)$ & \\
\hline \multicolumn{4}{|l|}{ Asthma } \\
\hline Yes & 14 & 5460 (2885-8035) & 0.675 \\
\hline No & 607 & 4504 (4269-4740) & \\
\hline \multicolumn{4}{|c|}{ Admission to intensive care unit } \\
\hline Yes & 123 & 7014 (6275-7753) & $<0.001$ \\
\hline No & 498 & $3911(3713-4110)$ & \\
\hline Thromboly & & & \\
\hline Yes & 25 & 7966 (6382-9550) & $<0.001$ \\
\hline No & 596 & $4382(4151-4613)$ & \\
\hline Year & & & \\
\hline 2010 & 362 & 4699 (4387-5011) & 0.034 \\
\hline 2008 & 259 & $4284(3923-4646)$ & \\
\hline Hospital & & & \\
\hline $\mathrm{H} 1$ & 127 & 3343 (3037-3343) & $<0.001$ \\
\hline $\mathrm{H} 2$ & 108 & $3659(3172-4146)$ & \\
\hline H3 & 84 & 3964 (3415-4512) & \\
\hline $\mathrm{H} 4$ & 24 & $4285(3176-5393)$ & \\
\hline $\mathrm{H} 5$ & 35 & $4741(3871-5610)$ & \\
\hline H6 & 31 & 5302 (4072-6532) & \\
\hline H7 & 85 & $5483(4761-6205)$ & \\
\hline $\mathrm{H} 8$ & 38 & $5711(4901-6521)$ & \\
\hline H9 & 74 & 6049 (5209-6890) & \\
\hline $\mathrm{H} 10$ & 15 & $6270(3099-9441)$ & \\
\hline
\end{tabular}

DVT: deep venous thrombosis; PE: pulmonary embolism; COPD: chronic obstructive pulmonary disease.

\#: comparisons from baseline (age < 40 years) with Bonferroni's correction for the multiple comparisons. 
TABLE 6 Multivariable linear regression analysis of predictors of log-transformed total costs as dependent variable

\begin{tabular}{lcc} 
Predictors & Relative increase $(95 \% \mathbf{C l})$ in cost & p-value \\
\hline Age group & $1.07(1.04-1.10)$ & 0.001 \\
Chronic pulmonary heart disease & $1.18(1.07-1.29)$ & 0.002 \\
Heart failure & $1.52(1.25-1.85)$ & 0.001 \\
Intensive care unit & $1.49(1.31-1.69)$ & 0.001 \\
Thrombolysis & $1.54(1.27-1.88)$ & 0.001 \\
Type of hospital & $1.06(1.04-1.07)$ & 0.001 \\
\hline
\end{tabular}

$p<0.0001$ for the model with $R^{2}=0.289$ for this six-variable model. Estimation of the parameters and the $95 \%$ confidence intervals was carried out using the bootstrap technique.

TABLE 7 Univariable linear regression analysis of predictors of log-transformed components of cost according to age groups: $<40,40-49,50-59,60-69,70-79$ and $\geqslant 80$ years

\begin{tabular}{|c|c|c|c|}
\hline & Patients n & Mean $(95 \% \mathrm{Cl})$ cost per stay EUR & p-value \\
\hline \multicolumn{4}{|c|}{ Nursing activity } \\
\hline$<40$ & 54 & $817(651-983)$ & \\
\hline $40-49$ & 65 & $1046(876-1216)$ & $0.114^{\#}$ \\
\hline $50-59$ & 52 & $1189(834-1544)$ & $0.739^{\#}$ \\
\hline $60-69$ & 100 & 1097 (896-1298) & $0.325^{\#}$ \\
\hline $70-79$ & 157 & 1407 (1256-1558) & $<0.001^{\#}$ \\
\hline$\geqslant 80$ & 193 & $1318(1183-1452)$ & $<0.001^{\#}$ \\
\hline \multicolumn{4}{|c|}{ ANOVA for linear trend $p<0.0001$} \\
\hline \multicolumn{4}{|c|}{ Medical activity } \\
\hline$<40$ & 54 & $980(846-1114)$ & \\
\hline $40-49$ & 65 & $1127(994-1260)$ & $0.574^{\#}$ \\
\hline $50-59$ & 52 & $1099(916-1281)$ & $0.980^{\#}$ \\
\hline $60-69$ & 100 & 1245 (1087-1402) & $0.157^{\#}$ \\
\hline $70-79$ & 157 & $1456(1323-1590)$ & $<0.001^{\#}$ \\
\hline$\geqslant 80$ & 193 & 1469 (1354-1583) & $<0.001^{\#}$ \\
\hline \multicolumn{4}{|c|}{ ANOVA for linear trend $p<0.0001$} \\
\hline \multicolumn{4}{|c|}{ Pharmacy } \\
\hline$<40$ & 54 & $216(148-285)$ & \\
\hline $40-49$ & 65 & $388(168-608)$ & $0.658^{\#}$ \\
\hline $50-59$ & 52 & $362(213-511)$ & $0.212^{\#}$ \\
\hline $60-69$ & 100 & $348(244-452)$ & $0.624^{\#}$ \\
\hline $70-79$ & 157 & $415(327-502)$ & $0.003^{\#}$ \\
\hline$\geqslant 80$ & 193 & $303(238-367)$ & $0.131^{\#}$ \\
\hline \multicolumn{4}{|c|}{ ANOVA for linear trend $p=0.315$} \\
\hline \multicolumn{4}{|c|}{ Administrative cost } \\
\hline$<40$ & 54 & 225 (192-257) & \\
\hline $40-49$ & 65 & $262(228-295)$ & $0.586^{\#}$ \\
\hline $50-59$ & 52 & $282(223-340)$ & $0.980^{\#}$ \\
\hline $60-69$ & 100 & $270(237-303)$ & $0.733^{\#}$ \\
\hline $70-79$ & 157 & $332(298-366)$ & $<0.001^{\#}$ \\
\hline$\geqslant 80$ & 193 & 327 (299-355) & $<0.001^{\#}$ \\
\hline ANOVA & & & \\
\hline \multicolumn{4}{|c|}{ Hospitalisation accommodation } \\
\hline$<40$ & 54 & 463 (393-533) & \\
\hline $40-49$ & 65 & $610(532-687)$ & $0.002^{\#}$ \\
\hline $50-59$ & 52 & 618 (505-730) & $0.107^{\#}$ \\
\hline $60-69$ & 100 & $637(549-726)$ & $0.044^{\#}$ \\
\hline $70-79$ & 157 & 805 (727-883) & $<0.001^{\#}$ \\
\hline$\geqslant 80$ & 193 & $833(755-910)$ & $<0.001^{\#}$ \\
\hline ANOVA & & & \\
\hline
\end{tabular}

\footnotetext{
\#: comparisons from baseline lage <40 years) with Bonferroni's correction for the multiple comparisons.
} 
shown by the broad IQR and the CVs of costs of patients within each SOI level. CVs were in the same range from $46 \%$ to $56 \%$ between SOI levels, suggesting that the magnitude of variability of costs was comparable across the four SOI classes. Whether this range of CVs is acceptable to consider that costs of patients within a SOI are homogenous is a matter of controversy [11].

Our study shows that the main cost components in both inlier and outlier patients were medical procedures, nursing activities and hospitalisation accommodation costs. Data are scarce regarding cost components associated with care of hospitalised patients with the primary diagnosis of PE. In a recent US study, the largest components of PE care expenses were nursing costs, followed by pharmacy and radiology costs [8]. Surprisingly, in our study, the greatest dispersion of values of cost was observed for pharmaceutical products in all SOI classes. This suggests that there was some heterogeneity in practice and in the use of antithrombotic agents despite the fact that the treatment of PE is well established and standardised in clinical guidelines.

A secondary study aim was to identify independent variables available at admission that could predict the costs. Six independent predictors of costs were identified: patient's age, chronic pulmonary heart disease, heart failure, admission to ICU, initial thrombolysis treatment and type of hospital. This multivariable model explained $29 \%$ of the variation in costs $\left(\mathrm{R}^{2}=0.289, \mathrm{p}<0.0001\right)$. This means that the combination of independent predictors in our regression model only predicted $\sim 30 \%$ of the variance of the costs. This may not be surprising as values of $\mathrm{R}^{2}$ are often of the order of $\leqslant 20 \%$ on regression analyses of utilisation data, low values of $\mathrm{R}^{2}$ indicating that the regression analysis cannot predict well for an individual patient [16]. However, our objective was to predict the average cost for a group of individuals. We show that costs were higher in older patients. Few data exist on the hospitalisation costs of PE according to patient's age. In a study using data from a database containing claim information from managed care organisations, average costs were $\sim 25 \%$ higher in patients aged $\geqslant 70$ years compared with the whole population [6]. Our results are consistent with those of that study. We show that the main cost components, including costs of nursing activities, medical procedures and hospitalisation accommodation, increased with age groups. This suggests that higher costs among older patients may be explained by the severity of comorbid conditions and complications among these patients.

Among the strengths of the current analysis, we included consecutive patients with a primary diagnosis of $\mathrm{PE}$ and admitted through the emergency department. This choice was made to isolate a homogeneous population with the diagnosis of PE that justified the hospitalisation. Our patients were comparable with those admitted in Belgium. The proportion of patients in each severity of illness subclass related to the APR DRG and the lengths of stay according to the degree of severity index of the APR DRG were quite similar to those reported in the national database concerning the APR DRG [6].

Finally, we should recognise the limitations of our study. First, identifying patients by using ICD-9-CM codes may lead to inaccuracies in diagnosis owing to imperfections in hospital coding procedures. However, $\mathrm{PE}$ is a disease with a clear definition, and the primary diagnosis assignment was made by experienced coders using rigid ICD-9-CM coding guidelines and conventions. Prior studies using medical chart review as the reference have shown that $94-96 \%$ of patients with ICD-9-CM codes for VTE had objectively documented disease [17-19]. Second, our work is based on a retrospective analysis of inpatient hospital discharge minimal clinical summaries and cost records. We had no access to medical charts and we could therefore not include in our analyses clinical variables such as signs and symptoms of PE present at admission that could be possible predictors of costs. Third, health service use and associated costs in our clinical setting may not be representative of other countries with different healthcare systems. However, our cost estimations are comparable with those reported in a recent Spanish study [4].

In conclusions, cost estimates obtained by aggregating all cost drivers per individual patient hospitalised with a primary diagnosis of PE were strongly related to the APR DRG SOI classes. Medical procedures, nursing activities and hospitalisation accommodation were the main cost drivers accounting for $\sim 70 \%$ of the total cost. Six independent predictors of costs were identified: patient's age, comorbidities of chronic pulmonary heart disease, heart failure, admission to ICU, initial thrombolysis treatment and type of hospital. The three main cost drivers significantly increased with age groups.

\section{References}

1 Oger E. Incidence of venous thromboembolism: a community-based study in Western France. EPI-GETBP Study Group. Groupe d'Etude de la Thrombose de Bretagne Occidentale. Thromb Haemost 2000; 83: 657-660.

2 Goldhaber SZ, Elliott CG. Acute pulmonary embolism: part I: epidemiology, pathophysiology, and diagnosis. Circulation 2003; 108: 2726-2729.

3 Park B, Messina L, Dargon P, et al. Recent trends in clinical outcomes and resource utilization for pulmonary embolism in the United States: findings from the nationwide inpatient sample. Chest 2009; 136: 983-990.

4 de Miguel-Díez J, Jiménez-García R, Jiménez D, et al. Trends in hospital admissions for pulmonary embolism in Spain from 2002 to 2011. Eur Respir J 2014; 44: 942-950. 
5 DeMonaco NA, Dang Q, Kapoor WN, et al. Pulmonary embolism incidence is increasing with use of spiral computed tomography. Am J Med 2008; 121: 611-617.

6 Spyropoulos AC, Lin J. Direct medical costs of venous thromboembolism and subsequent hospital readmission rates: an administrative claims analysis from 30 managed care organizations. J Manag Care Pharm 2007; 13: 475-486.

7 Merli G, Ferrufino C, Lin J, et al. Hospital-based costs associated with venous thromboembolism treatment regimens. J Thromb Haemost 2008; 6: 1077-1086.

8 Fanikos J, Rao A, Seger AC, et al. Hospital costs of acute pulmonary embolism. Am J Med 2013; 126: 127-132.

9 Lin J, Lingohr-Smith M, Kwong WJ. Incremental health care resource utilization and economic burden of venous thromboembolism recurrence from a U.S. payer perspective J Manag Care Pharm 2014; 20: 174-186.

10 Pirson M, Schenker L, Martins D, et al. What can we learn from international comparisons of costs by DRG? Eur J Health Econ 2013; 14: 67-73.

11 Pirson M, Delo C, Di Pierdomenico L, et al. Variability of nursing care by APR-DRG and by severity of illness in a sample of nine Belgian hospitals. BMC Nurs 2013; 12: 2-6.

12 Pilotto A, Scarcelli C, D'Ambrosio LP, et al. All patient refined diagnosis related groups: a new administrative tool for identifying elderly patients at risk of high resource consumption. J Am Geriatr Soc 2005; 53: 167-168.

13 Shah AN, Vail TP, Taylor D, et al. Comorbid illness affects hospital costs related to hip arthroplasty: quantification of health status and implications for fair reimbursement and surgeon comparisons. J Arthroplasty 2004; 19: 700-705.

14 Lavernia CJ, Laoruengthana A, Contreras JS, et al. All-Patient Refined Diagnosis-Related Groups in primary arthroplasty. J Arthroplasty 2009; 24: 6 Suppl., 19-23.

15 Adrados M, Lajam C, Hutzler L, et al. The effect of severity of illness on total joint arthroplasty costs across New York state hospitals: an analysis of 172,738 cases. J Arthroplasty 2015; 30: 12-14.

16 Diehr P, Yanez D, Ash A, et al. Methods for analyzing health care utilization and costs. Annu Rev Public Health 1999; 20: 125-144.

17 White RH, Zhou H, Romano PS. Length of hospital stay for treatment of deep venous thrombosis and the incidence of recurrent thromboembolism. Arch Intern Med 1998; 158: 1005-1010.

18 White RH, Gettner S, Newman JM, et al. Predictors of rehospitalization for symptomatic venous thromboembolism after total hip arthroplasty. N Engl J Med 2000; 343: 1758-1764.

19 Murin S, Romano PS, White RH. Comparison of outcomes after hospitalization for deep venous thrombosis or pulmonary embolism. Thromb Haemost 2002; 88: 407-414. 